\title{
Identification of inhomogeneous residual stress state in elastic cylinder within the framework of plane strain
}

\author{
Ivan Victorovich Bogacheva ${ }^{\mathrm{a}}$, Vladimir Vladimirovich Dudarev ${ }^{\mathrm{b}^{*}}$, \\ Rostislav Dmitrievich Nedin ${ }^{d}$ and Alexander Ovanesovich Vatulyan ${ }^{d}$ \\ Department of Theory of Elasticity, Faculty of Mathematics, Mechanics and Computer Sciences, \\ Southern Federal University, 8a, Mil'chakova Street, 344090, Rostov-on-Don, Russia \\ abogachev89@yandex.ru, bdudarev_vv@mail.ru, ${ }^{c} r d n 90 @ b k . r u,{ }^{d}$ vatulyan@math.rsu.ru
}

Keywords: Inhomogeneous residual stress, acoustic sounding method, cylinder, inverse problem, iterative process.

Abstract. The problem on radial oscillations of an elastic cylinder with inhomogeneous residual stress (RS) is considered. Two acoustic techniques of RS reconstructing are suggested. Within the framework of the first method, a set of radial displacement values is assumed to be known, while the frequency is fixed. Within the framework of the second method, the radial displacement value at the outer radius is assumed to be known for a set of frequencies. The examples of numerical identification experiments are presented.

\section{Problem statement}

The motion equations, constitutive relations, and mixed boundary conditions describing steadystate vibrations of isotropic body under residual stress state (RSS) have form [1,2]:

$$
\begin{aligned}
& \nabla \cdot \underline{\underline{T}}+\rho \omega^{2} \underline{u}=0, \underline{\underline{T}}=\underline{\underline{\sigma}}+\underline{\underline{\sigma}}^{0} \cdot \nabla \underline{u}, \nabla \cdot \underline{\underline{\sigma}}^{0}=0, \underline{\underline{\sigma}}=\lambda \underline{\underline{E}} \operatorname{tr} \underline{\underline{\varepsilon}}+2 \mu \underline{\underline{\varepsilon}}, \underline{\underline{\varepsilon}}=0.5\left(\nabla \underline{u}+\nabla \underline{u}^{T}\right), \\
& \left.\underline{n} \cdot \underline{\underline{T}}\right|_{S_{\sigma}}=\underline{p},\left.\underline{u}\right|_{S_{u}}=0
\end{aligned}
$$

where $\nabla=\underline{e}_{r} \partial / \partial r+\underline{e}_{\varphi} r^{-1} \partial / \partial \varphi+\underline{e}_{z} \partial / \partial z, \rho$ is body's density, $\omega$ is vibration frequency, $\underline{u}$ is displacement vector, $\underline{\underline{T}}$ is the first nonsymmetrical Piola-Kirchhoff stress tensor, $\underline{\underline{\sigma}}^{0}$ is the RS tensor, $\lambda, \mu$ are the Lamé constants, $\underline{\underline{\varepsilon}}$ is strain tensor, body force is absent. Let us represent the expressions of displacement gradient $\nabla \underline{u}$, tensors $\underline{\underline{T}}$ and $\underline{\underline{\sigma}}^{0}$ subject to the plane deformation $\underline{u}\left(u_{r}(r, \varphi), u_{\varphi}(r, \varphi)\right.$, const $)$ in the polar coordinate system $(r, \varphi)$ :

$$
\begin{aligned}
& \underline{\underline{T}}=T_{r r} \underline{e}_{r} \underline{e}_{r}+T_{r \varphi} \underline{e}_{r} \underline{e}_{\varphi}+T_{\varphi r} \underline{e}_{\varphi} \underline{e}_{r}+T_{\varphi \varphi} \underline{e}_{\varphi} \underline{e}_{\varphi}, \underline{\underline{\sigma}}^{0}=\sigma_{r r}^{0} \underline{e}_{r} \underline{e}_{r}+\sigma_{r \varphi}^{0} \underline{e}_{r} \underline{e}_{\varphi}+\sigma_{\varphi r}^{0} \underline{e}_{\varphi} \underline{e}_{r}+\sigma_{\varphi \varphi}^{0} \underline{e}_{\varphi} \underline{e}_{\varphi}, \\
& \nabla \underline{u}=\frac{\partial u_{r}}{\partial r} \underline{e}_{r} \underline{e}_{r}+\frac{\partial u_{\phi}}{\partial r} \underline{e}_{r} \underline{e}_{\phi}+\frac{1}{r}\left(\frac{\partial u_{r}}{\partial \phi}-u_{\phi}\right) \underline{e}_{\phi} \underline{e}_{r}+\frac{1}{r}\left(\frac{\partial u_{\phi}}{\partial \phi}+u_{r}\right) \underline{e}_{\phi} \underline{e}_{\phi} .
\end{aligned}
$$

As a particular practical example of the statement presented let us consider axisymmetric problem on steady-state vibration of a cylindrical region (inner radius $r_{1}>0$, outer radius $r_{2}>r_{1}$ ). Vibrations are caused by uniformly-distributed load $\underline{p}=-p \underline{e}_{r}$ applied to the outer contour $r=r_{2}$. The boundary conditions take form

$$
\left.\left(T_{r r} \underline{e}_{r}+T_{r \varphi} \underline{e}_{\varphi}\right)\right|_{r=r_{2}}=\underline{p},\left.\left(T_{r r} \underline{e}_{r}+T_{r \phi} \underline{e}_{\phi}\right)\right|_{r=r_{1}}=0 .
$$


Assume that the only nonzero components of the RS tensor are two components $\sigma_{r r}^{0}=\sigma_{r r}^{0}(r) \neq 0, \sigma_{\varphi \varphi}^{0}=\sigma_{\varphi \varphi}^{0}(r) \neq 0$. Due to load symmetry and region geometry, the nonzero component of radial displacement $u_{r}$ is a function of radius $r$ only:

$$
u_{r}=u(r), u_{\phi}=0
$$

On the basis of the general formulas (1)-(4) let us express the components of the PiolaKirchhoff tensor and the motion equation:

$$
T_{r r}=\sigma_{r r}+\frac{d u}{d r} \sigma_{r r}^{0}, T_{r \varphi}=0, T_{\varphi \varphi}=\sigma_{\varphi \varphi}+\frac{1}{r} u \sigma_{\varphi \varphi}^{0}, T_{\varphi r}=0, \frac{d T_{r r}}{d r}+\frac{T_{r r}-T_{\phi \phi}}{r}+\rho \omega^{2} u=0
$$

The equilibrium condition for the RS tensor $\underline{\underline{\sigma}}^{0}$ from (1) take form of relation binding together the components $\sigma_{r r}^{0}$ and $\sigma_{\varphi \varphi}^{0}$ :

$$
\sigma_{\phi \phi}^{0}=r \frac{d \sigma_{r r}^{0}}{d r}+\sigma_{r r}^{0}
$$

Taking into account the relation obtained, let us write the motion equation in terms of displacement and the boundary conditions:

$$
\begin{aligned}
& \left(\lambda+2 \mu+\sigma_{r r}^{0}\right) \frac{d^{2} u}{\partial r^{2}}+\left(\frac{d \sigma_{r r}^{0}}{d r}+\frac{1}{r}\left(\lambda+2 \mu+\sigma_{r r}^{0}\right)\right) \frac{d u}{d r}+\left(\rho \omega^{2}-\frac{1}{r^{2}}\left(\lambda+2 \mu+r \frac{d \sigma_{r r}^{0}}{d r}+\sigma_{r r}^{0}\right)\right) u=0,(9) \\
& \left.\left(\left(\lambda+2 \mu+\sigma_{r r}^{0}\right) \frac{d u}{\partial r}+\frac{\lambda}{r} u\right)\right|_{r=r_{2}}=-p,\left.\left(\left(\lambda+2 \mu+\sigma_{r r}^{0}\right) \frac{d u}{\partial r}+\frac{\lambda}{r} u\right)\right|_{r=r_{1}}=0
\end{aligned}
$$

The Lamé parameters $\lambda, \mu$ in the expressions above are assumed to be constants. Next, let us introduce dimensionless parameters and functions: $p^{*}=-p r_{2} /(\lambda+2 \mu), \kappa^{2}=\rho \omega^{2} r_{2}^{2} /(\lambda+2 \mu)$ is a parameter characterizing vibration frequency, $\xi \in\left[\xi_{0}, 1\right]$ is the dimensionless radial coordinate, $\xi_{0}=r_{1} / r_{2}, k=\lambda /(\lambda+2 \mu), g(\xi)=\sigma_{r r}^{0} / \max _{\xi \in\left[\xi_{0}, 1\right]}\left|\sigma_{r r}^{0}\right|$ is a function describing the law of variation of the component $\sigma_{r r}^{0}, \tau=\max _{\xi \in\left[\xi_{0}, 1\right]}\left|\sigma_{r r}^{0}\right| /(\lambda+2 \mu)$ is a parameter characterizing the level of RSS, $u(r)=r_{2} U(\xi)$. The motion equation and the boundary conditions will take form

$$
\begin{aligned}
& (1+\tau g) U^{\prime \prime}+\left(\tau g^{\prime}+\frac{1+\tau g}{\xi}\right) U^{\prime}-\left(\frac{1+\tau g}{\xi^{2}}+\frac{\tau g^{\prime}}{\xi}-\kappa^{2}\right) U=0, \\
& (1+\tau g) U^{\prime}+\left.k \frac{U}{\xi}\right|_{\xi=1}=p^{*},(1+\tau g) U^{\prime}+\left.k \frac{U}{\xi}\right|_{\xi=\xi_{0}}=0 .
\end{aligned}
$$

The Eq. (11) is a $1^{\text {st }}$ order differential equation with variable coefficients. A solution of the boundary problem (11)-(12) generally may be found only numerically. One of the possible methods to solve it is the shooting method. To realize it, the problem is reduced to a system of two differential equations for two functions $y(\xi)=U^{\prime}(\xi)$ and $U(\xi)$. The numerical solution is obtained in the package Maple using standard procedures. In case of homogeneous $\operatorname{RSS}(g(\xi)=1)$, an influence of the parameter $\tau$ on frequency response function (FRF) revealed that the most sufficient effect appears in close vicinity of resonances and for higher values of natural frequencies. 


\section{Inverse problem}

The inverse problem for the object considered is to identify the function $g(\xi)$ from some additional data on FRF. Let us view two approaches.

In frames of the first one, as additional information we assume the nodal values of the displacement function $U\left(\xi_{i}, \kappa\right), i=1 . . N$ in a finite set of points $\xi_{i} \in\left[\xi_{0}, 1\right]$ for a fixed parameter $\kappa \in\left[\kappa_{-}, \kappa_{+}\right]$. At that, the motion equation (11) may be considered as $1^{\text {st }}$ order differential equation with variable coefficients with regard to the unknown function $g(\xi)$ :

$$
\left(U^{\prime}-\frac{U}{\xi}\right) \tau g^{\prime}+\left(U^{\prime \prime}+\frac{U^{\prime}}{\xi}-\frac{U}{\xi^{2}}\right) \tau g+U^{\prime \prime}+\frac{U^{\prime}}{\xi}-\frac{U}{\xi^{2}}+\kappa^{2} U=0,(1+\tau g) U^{\prime}+\left.k \frac{U}{\xi}\right|_{\xi=1}=p^{*}
$$

The solution of this problem may be rewritten as follows:

$$
g(\xi)=(F(\xi)+C) \cdot \Phi(\xi)
$$

where

$$
\begin{aligned}
& F(\xi)=\int_{\xi}^{1} \frac{B(\xi)}{\Phi(\xi)} d \xi, \Phi(\xi)=\frac{1}{\tau} \exp \left(\int_{\xi}^{1} A(\xi) d \xi\right), C=\frac{p^{*}-k U(1)}{U^{\prime}(1)}-1 \\
& A(\xi)=\frac{U^{\prime \prime}(\xi)+\frac{U^{\prime}(\xi)}{\xi}-\frac{U(\xi)}{\xi^{2}}}{U^{\prime}(\xi)-\frac{U(\xi)}{\xi}}, B(\xi)=\frac{U^{\prime \prime}(\xi)+\frac{U^{\prime}(\xi)}{\xi}+\left(\kappa^{2}-\frac{1}{\xi^{2}}\right) \cdot U(\xi)}{U^{\prime}(\xi)-\frac{U(\xi)}{\xi}} .
\end{aligned}
$$

It is evident from the Eq. (16) that the solution of the Eq. (14) does not exist or nonunique if $s(\xi, \kappa)=U^{\prime}(\xi, \kappa)-U(\xi, \kappa) / \xi=0$. The calculations showed that there are some points $\left(\xi_{*}, \kappa_{*}\right)$ at frequency ranges above the $1^{\text {st }}$ resonance frequency in which the function $s(\xi, \kappa)$ may become zero. Moreover, a number of such points increases with selection of higher frequency ranges. Thus, it is necessary to implement the inverse problem solving in accordance with formulas (14)-(16) for a given frequency below the $1^{\text {st }}$ resonance.

Another important aspect of implementation of such an approach is a problem of approximation of the displacement function $U(\xi)$ by a given set of its nodal values $U\left(\xi_{i}, \kappa\right), i=1 . . N$, and further calculation of its first and second derivatives which is occurred using spline-interpolations. The computational experiments on a recovering of monotonic and non-monotonic functions $g(\xi)$ are carried out in the package Maple with using splines of the fifth degree. The most precise reconstruction is typical for thin-walled cylinders $\left(\xi_{0}>0.7\right)$. For instance, a relative error of a reconstruction of the function $g(\xi)=6^{(14-5 \xi)}$ for $\xi_{0}=0.9, k=0.4, p^{*}=1, \kappa=0.6, \tau=10^{-3}$, $N=30$ was less than $3 \%$. Also let us note that for a successful reconstruction of RSS of $10^{-3}$ level, the accuracy of the input data $\left(U\left(\xi_{i}, \kappa\right), \kappa, k, p^{*}\right)$ should be set up to $10^{-4}$.

In the framework of the second approach to reconstruction of inhomogeneous RSS, as additional information we assume the given values of the displacement at the outer radius $U(1, \kappa)=d(\kappa)$ in a finite set of frequencies $\kappa \in\left[\kappa_{-}, \kappa_{+}\right]$. The implementation of this approach is based on a constructing of an iterative process $[3,4]$. To derive this relation, we shall use the linearization method. Let us rewrite the motion equation and the boundary conditions (11)-(12) in the form: 


$$
\begin{aligned}
& \xi\left((1+\tau g) U^{\prime}\right)^{\prime}+(1+\tau g) U^{\prime}-\frac{(1+\tau g) U}{\xi}-\tau g^{\prime} U+\xi \kappa^{2} U=0, \\
& (1+\tau g(1)) U^{\prime}(1)+k U(1)=p^{*}, \xi_{0}\left(1+\tau g\left(\xi_{0}\right)\right) U^{\prime}\left(\xi_{0}\right)+k U\left(\xi_{0}\right)=0 .
\end{aligned}
$$

According to the linearization method, let us put

$$
g=\varepsilon g_{1}, U=U_{0}+\varepsilon U_{1},
$$

where $\varepsilon$ is formal parameter. Let us substitute (19) into the motion equation and the boundary condition. Next, by writing down the problems corresponding to the zeroth and first degrees of the parameter $\varepsilon$, and by occurring integral transformations, we get the following operator relation:

$$
\begin{aligned}
& \int_{\xi_{0}}^{1} \tau g_{1}(\xi)\left(\xi U_{0}^{\prime 2}(\xi, \kappa)+U_{0}^{2}(\xi, \kappa) / \xi\right) d \xi+\int_{\xi_{0}}^{1} \tau g_{1}^{\prime}(\xi) U_{0}^{2}(\xi, \kappa) d \xi=p^{*}\left(U_{0}(1, \kappa)-d(\kappa)\right) \\
& \kappa \in\left[\kappa_{-}, \kappa_{+}\right]
\end{aligned}
$$

This relation may be treated as integro-differential equation for sought-for correction of radial RSS component $g_{1}(\xi)$ with the displacement function $d(\kappa)=U(1, \kappa)$ measured. The Eq. (20) $U_{0}(\xi, \kappa)$ contains the displacement function calculated at previous step. Note that the Eq. (20) can also be transformed into the integral form:

$$
\begin{aligned}
& \int_{\xi_{0}}^{1} \tau g_{1}(\xi)\left(\xi U_{0}^{\prime}(\xi, \kappa)-U_{0}(\xi, \kappa)\right)^{2} / \xi d \xi+\tau g_{1}(1) U_{0}^{2}(1, \kappa)-\tau g_{1}\left(\xi_{0}\right) U_{0}^{2}\left(\xi_{0}, \kappa\right)= \\
& =p^{*}\left(U_{0}(1, \kappa)-d(\kappa)\right), \kappa \in\left[\kappa_{-}, \kappa_{+}\right] .
\end{aligned}
$$

The solving of this equation is implemented numerically by discretization of integral operators and further using of the A.N. Tikhonov regularization method [5]. To organize an iterative process, the initial approximation for $g(\xi)$ was searched in a class of linear functions $g(\xi)=a+b \xi$. Values of the coefficients $a, b$ are determined from the minimizing of residual functional at a compact set:

$$
G=\int_{\kappa_{-}}^{\kappa_{+}}|\tilde{U}(1, \kappa)-d(\kappa)|^{2} d \kappa .
$$

where $\tilde{U}(1, \kappa)$ is a function of displacement at the outer radius of the ring for a linear law of variation of the function $g(\xi)$. The compact set considered is a set in a space of real parameters $(a, b)$ which is built from a condition of boundedness of the sought-for function $\lg (\xi) \mid \leq M$.

When the quadrature trapezoidal rule was used, the computational experiments showed that the approach proposed may be successfully employed for a recovering of monotonic and nonmonotonic functions $g(\xi)$. The relative error for monotonic functions does not exceed $4 \%$, and for non-monotonic $-10 \%$, when frequency range is selected between the $1^{\text {st }}$ and the $2^{\text {nd }}$ resonance frequencies. As well as for the first approach, the most precise reconstruction is typical for thinwalled cylinders $\left(\xi_{0}>0.7\right)$. For example, when reconstructing the function $g(\xi)=\xi^{-7}-1$, the maximum relative error was $2.5 \%$ for the following parameters values: $\xi_{0}=0.9, \tau=10^{-3}$, $\kappa \in[2,5], k=0.4, p^{*}=1$. 


\section{Summary}

Two approaches to a reconstruction of inhomogeneous RSS in a cylinder under plane deformation are proposed. In frames of each approach, the analysis of computational experiments on determining RSS laws of variation is carries out.

The research is done with the support of grants of the Russian Foundation of Basic Research (project codes: 13-01-00196-A, 14-01-31393 mol_a).

\section{References}

[1] E. Trefftz, Zur theorie der stabilitat des elastischen gleichgewichts, ZAMM: Z. angew. Math. Mech. V. 12, 2. (1933) 160-165.

[2] A. N. Guz, Three-dimensional theory of elastic stability under finite subcritical deformations, J. Appl. Mech. V. 8, 12. (1972) 25-44.

[3] V.V. Dudarev, A.O. Vatulyan, On restoring of the pre-stressed state in elastic bodies, ZAMM: Z. angew. Math. Mech. V. 91, 6. (2011) 485-492.

[4] R.D. Nedin, A.O. Vatulyan, Concerning one approach to the reconstruction of heterogeneous residual stress in plate, ZAMM: Z. angew. Math. Mech. V. 94, 1-2 (2014) 142-149.

[5] R.D. Nedin, A.O. Vatulyan, Inverse Problem of Non-homogeneous Residual Stress Identification in Thin Plates, Int. J. Solids Struct. 50 (2013) 2107-2114. 